\title{
Origin of symmetry-forbidden high-order harmonic generation in the time-dependent Kohn-Sham formulation
}

\author{
Xiaoning Zang, ${ }^{1}$ Ce Shang $\odot,{ }^{1}$ Pengcheng Zhang, ${ }^{2}$ Yalei Zhu, ${ }^{3}$ Zhiyong Zhu, ${ }^{4}$ and Udo Schwingenschlögl ${ }^{1, *}$ \\ ${ }^{1}$ Physical Sciences and Engineering Division (PSE), King Abdullah University of Science and Technology (KAUST), Thuwal 23955-6900, \\ Saudi Arabia \\ ${ }^{2}$ Department of Chemistry, Xiamen University, Xiamen 361005, China \\ ${ }^{3}$ Department of Physics, National University of Defense Technology, Changsha 410073, China \\ ${ }^{4}$ KAUST Supercomputing Laboratory (KSL), King Abdullah University of Science and Technology (KAUST), \\ Thuwal 23955-6900, Saudi Arabia
}

(Received 26 April 2020; accepted 23 March 2021; published 12 April 2021)

\begin{abstract}
We find, for two-electron closed-shell systems $\left(\mathrm{H}_{2}\right.$ molecule, He atom), symmetry-forbidden peaks in the high-order harmonic spectra obtained by the time-dependent Kohn-Sham equations, and clarify their origin. It turns out that fixation of the number of Kohn-Sham orbitals and their occupations gives rise to unphysical transition paths and, therefore, incorrect populations of the one-electron excited states, which leads to evenorder harmonics in systems with inversion symmetry. We show that the time-dependent natural Kohn-Sham and time-dependent configuration interaction equations do not suffer from this shortcoming.
\end{abstract}

DOI: 10.1103/PhysRevA.103.043106

\section{INTRODUCTION}

Since the first observation of high-order harmonic generation (HHG) in gases [1], the field has developed tremendously [2]. It is now well understood in terms of the semiclassical three-step model $[3,4]$ and the more rigorous Lewenstein model [5], which shows that the cutoff photon energy is proportional to the intensity and wavelength of the driving laser. To extend the cutoff, the duration of the laser pulse can be decreased so that the intensity is increased [6,7]. A pulse as short as $5 \mathrm{fs}$, approaching a single cycle, has been employed in Ref. [8] to generate a $0.5 \mathrm{keV}$ x-ray harmonic. However, high intensity leads to strong ionization and therefore decoherence, which limits the usability of such harmonics [9]. Alternatively, the wavelength of the laser can be increased [10]. Wavelengths ranging from 1.3 to $2.1 \mu \mathrm{m}$ [11-17] and even $3.9 \mu \mathrm{m}$ [18] have been used to obtain cutoffs around $1 \mathrm{keV}$. Recent experimental [19-23] and theoretical [24-32] efforts focus on HHG in solids. HHG has vast applications in modern physics, such as high-resolution core level [33] and plasma [34] spectroscopy, x-ray fluorescence analysis [35], attosecond time-resolved measurement of electron dynamics [36-40], extraction of higher-order nonlinear electronic responses [41], measurement of Berry curvatures [42], and characterization of topological phase transitions [43-46].

Numerical methods have been developed to simulate the process of HHG [47]. Among them, real-time time-dependent density functional theory was deemed to combine appropriate accuracy with acceptable computational cost for large systems. However, we find strong even-order harmonics in two-electron closed-shell systems with inversion symmetry

\footnotetext{
*udo.schwingenschlogl@kaust.edu.sa
}

using the time-dependent Kohn-Sham (TDKS) equations; see Fig. 1.

Theoretical works employing simplified models have addressed the failure to obtain clean odd-order harmonics in periodic systems. In semimetals, disturbed interference can generate a white spectrum that masks the odd-order harmonics [48], high-order harmonics can be blurred by elastic and inelastic scattering from impurities and phonons, respectively [49], inversion symmetry breaking can enhance even-order harmonics to the strength of odd-order harmonics [50], and long dephasing times can bury odd-order harmonics in strong background noise [51]. Out of these mechanisms, only a lack of inversion symmetry could explain our observations; however, the considered systems possess inversion symmetry. Due to the apparent absence of physical reasons, we consider a possible deficiency of the TDKS equations to explain the appearance of even-order harmonics. This idea is also inspired by the fact that unphysical two-electron excited states inherent to the TDKS equations, due to fixation of the number of KS orbitals and their occupations, lead to wrong electron density and, therefore, failure to describe resonant Rabi oscillations [52]. However, when a HHG spectrum is calculated, the system is not driven far away from the ground state so that two-electron excited states can be neglected.

In this paper, we demonstrate that the generation of symmetry-forbidden even-order harmonics is caused by incorrect populations of one-electron excited states when the TDKS equations are used. Additionally, two-electron excited states alter these populations by opening unphysical transition paths. This conclusion is further supported by comparison to HHG spectra obtained by time-dependent natural Kohn-Sham (TDNKS) and time-dependent configuration interaction (TDCI) simulations, and by fitting to the Lewenstein model. 

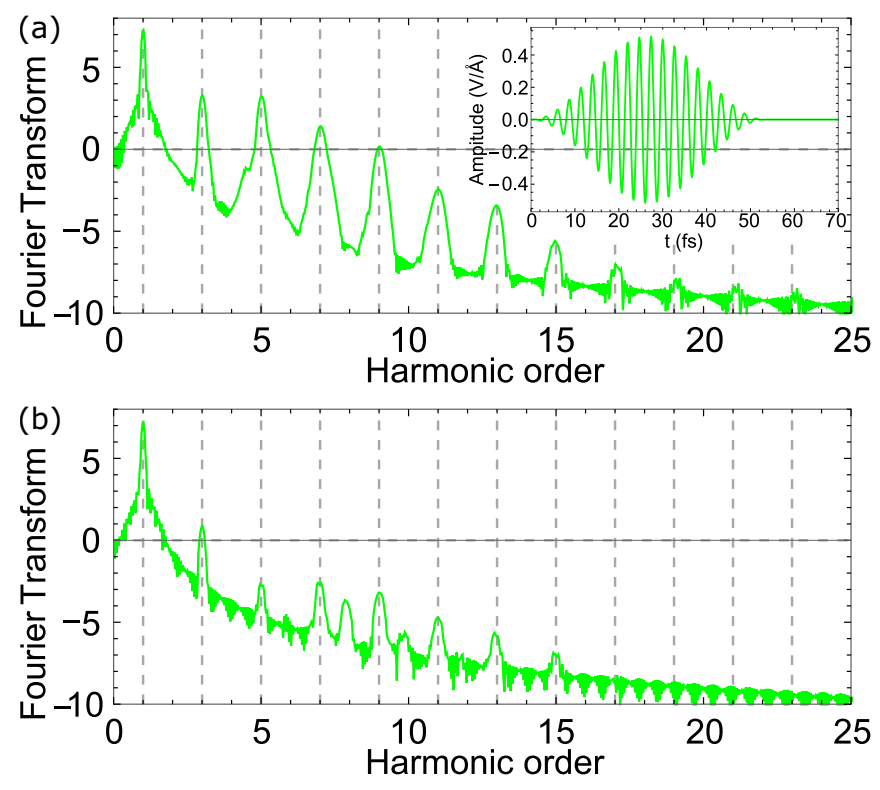

FIG. 1. TDKS HHG spectra of (a) a $\mathrm{H}$ atom (only odd-order harmonics) and (b) a $\mathrm{H}_{2}$ molecule (symmetry-forbidden even-order harmonics) for $\hbar \omega=1.55 \mathrm{eV}, \sigma=2.67 \times 10^{-14} \mathrm{~s}$, and $E_{0}=0.5$ $\mathrm{V} / \AA$. The inset shows the laser pulse.

\section{RESULTS AND DISCUSSION}

We use the TDKS implementation of the OCTOPUS code [53] and implement the TDNKS equations in this code (Appendix $\mathrm{A}$ ). We address a $\mathrm{H}$ atom as a prototypical one-electron system, a $\mathrm{H}_{2}$ molecule (optimized bond length of $0.74 \AA$ ) as a prototypical multielectron system with static correlation, and a $\mathrm{He}$ atom as a prototypical multielectron system without static correlation. For the $\mathrm{H}$ atom and $\mathrm{H}_{2}$ molecule (He atom), the spherical simulation box has a radius of 20 (10.5) $\AA$ and is discretized in increments of $0.2(0.15) \AA$. We employ Troullier-Martins pseudopotentials and the local density approximation (LDA) or Perdew-Burke-Ernzerhof (PBE) generalized gradient approximation. The time step is $\Delta t=2.4 \times 10^{-19} \mathrm{~s}$ in the TDCI and TDKS simulations, and $\Delta t=9.7 \times 10^{-20} \mathrm{~s}$ in the TDNKS simulations (occupations updated every $5 \Delta t$ while the laser pulse is applied). Tukey windows are used to eliminate spectral leakage (Appendix B).

The dipole approximation is employed for the light-matter interaction, $H_{1}=\vec{r} \cdot \vec{E}(t)$, with electric field

$$
\vec{E}(t)= \begin{cases}E_{0} \cos ^{2}\left(\frac{\pi(\sigma-t)}{2 \sigma}\right) \sin (\omega t) & \text { for } t \leqslant 2 \sigma \\ 0 & \text { for } t>2 \sigma,\end{cases}
$$

where $E_{0}$ and $\omega$ are the strength and frequency, respectively. The inset of Fig. 1 gives $\vec{E}(t)$ with $\hbar \omega=1.55 \mathrm{eV}, \sigma=2.67 \times$ $10^{-14} \mathrm{~s}$, and $E_{0}=0.5 \mathrm{~V} / \AA$. The TDKS HHG spectrum of the $\mathrm{H}$ atom only shows odd-order harmonics, as expected from the inversion symmetry of the $\mathrm{H}$ atom; see Fig. 1(a). The observation is confirmed for different sets of parameters of $\vec{E}(t)$. The TDKS HHG spectrum of the $\mathrm{H}_{2}$ molecule, on the other hand, also shows even-order harmonics, which physically should not appear due to the inversion symmetry of the $\mathrm{H}_{2}$ molecule; see Fig. 1(b). The TDKS equations therefore work well for the one-electron system, but fail for the multielectron system. As another peculiarity of the TDKS equations, it has been known for a long time that resonant Rabi oscillations can be generated only for one-electron systems. This recently has been explained by large populations of unphysical multielectron excited states that give rise to wrong electron density [52]. In the case of nonresonant Rabi oscillations, however, the TDKS equations work well for multielectron systems, since multielectron excited states play no relevant role. The fact that for our $\mathrm{H}_{2}$ molecule the two-electron excited states can also be neglected, as it stays close to the ground state, thus raises the question of why the TDKS equations still fail to describe the HHG.

To answer this question, we analyze the states contributing to the HHG spectrum. We benefit from the simplicity of the $\mathrm{H}_{2}$ molecule, as only the ground state, one-electron excited states, and two-electron excited states need to be considered. Describing the time-propagated multielectron state as a linear combination of these states, we have the TDCI equations (atomic units)

$$
\begin{aligned}
i \frac{\partial}{\partial t} C_{m n}(t)= & \left(\varepsilon_{m}+\varepsilon_{n}\right) C_{m n}(t)+\sum_{m^{\prime}=1}^{M} C_{m^{\prime} n}(t) d_{m m^{\prime}} \\
& +\sum_{n^{\prime}=1}^{M} C_{m n^{\prime}}(t) d_{n n^{\prime}},
\end{aligned}
$$

where $C_{m n}(t)$ is the coefficient of the Slater determinant $\left|\psi_{m} \bar{\psi}_{n}\right\rangle$ (with $\left|\psi_{m}\right\rangle$ and $\left|\bar{\psi}_{n}\right\rangle$ being the KS orbitals at $t=0$ for spin up and down, respectively), $\varepsilon_{m}$ is the eigenenergy of $\left|\psi_{m}\right\rangle, M$ is the number of KS orbitals, and $d_{m m^{\prime}}=\left\langle\psi_{m}|y| \psi_{m^{\prime}}\right\rangle$ is the transition dipole. For the initial conditions $C_{11}(t=0)=$ 1 and $C_{m n}(t=0)=0$ for $m \neq 1$ or $n \neq 1$, which correspond to the ground state, we can solve Eq. (2) for $C_{m n}(t)$ to obtain the dipole moment

$$
\begin{aligned}
d_{y}(t)= & \sum_{n=1}^{M} \sum_{m^{\prime}, m=1}^{M} C_{m^{\prime} n}^{*}(t) C_{m n}(t) d_{m^{\prime} m} \\
& +\sum_{m=1}^{M} \sum_{n^{\prime}, n=1}^{M} C_{m n^{\prime}}^{*}(t) C_{m n}(t) d_{n^{\prime} n} .
\end{aligned}
$$

See Appendix C for further details on Eqs. (2) and (3). The Fourier transform of $d_{y}(t)$ (we show the decadic logarithm in the following) gives rise to the HHG spectrum.

Using the time-propagated multielectron wave function $|\Psi(t)\rangle=\left|\psi_{1}(t) \bar{\psi}_{1}(t)\right\rangle$ obtained from the TDKS equations, where $\left|\psi_{1}(t)\right\rangle$ is the lowest $\mathrm{KS}$ orbital, the dipole moment is given by (Appendix C)

$$
d_{y}(t)=2\left\langle\psi_{1}(t)|y| \psi_{1}(t)\right\rangle .
$$

We can expand $\left|\psi_{1}(t)\right\rangle$ in terms of the KS orbitals at $t=0$ as $\left|\psi_{1}(t)\right\rangle=\sum_{i=1}^{M} a_{i}(t)\left|\psi_{i}\right\rangle$. Then, $C_{m n}(t)=a_{m}(t) a_{n}(t)$ [54] and Eq. (4) can be rewritten in the form of Eq. (3). Note, however, that the TDCI equations provide an exact solution of the KS system as long as $M$ is sufficiently large, whereas the TDKS equations are an approximation. Despite the equivalence of Eqs. (3) and (4), the calculated dipole moments thus will not be identical.

The TDKS and TDCI HHG spectra of a $\mathrm{H}_{2}$ molecule are shown in Fig. 2(a). First, we observe that the TDCI HHG spec- 

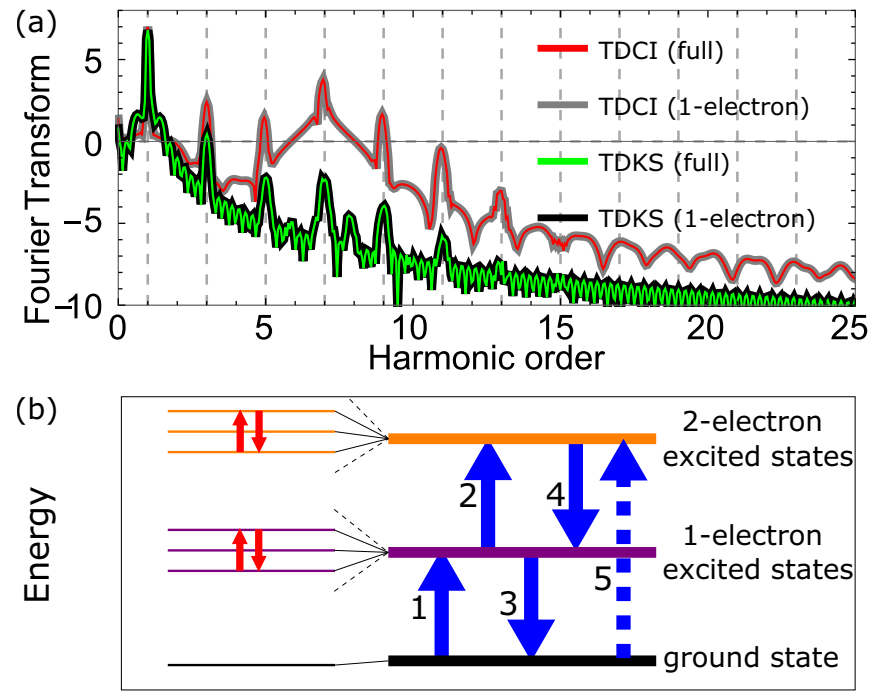

FIG. 2. (a) TDKS and TDCI HHG spectra of a $\mathrm{H}_{2}$ molecule for $\hbar \omega=1.55 \mathrm{eV}, \sigma=2.67 \times 10^{-14} \mathrm{~s}$, and $E_{0}=0.5 \mathrm{~V} / \AA$. The TDCI HHG spectra show no symmetry-forbidden peaks. Considering only one-electron excited states and considering all excited states results in perfectly matching HHG spectra (both for the TDKS and TDCI equations). (b) Red arrows: Transitions among one-electron excited states and among two-electron excited states. Solid blue arrows: Physical transitions (between ground state and one-electron excited state; between one-electron excited state and two-electron excited state). Dashed blue arrow: Unphysical transition (ground state to two-electron excited state).

tra have no even-order harmonics, in contrast to the TDKS HHG spectra. Second, for both methods, consideration of two-electron excited states in the calculation has virtually no influence on the HHG spectrum, implying that only the one-electron excited states matter. Note that the TDCI HHG spectrum without two-electron excited states is obtained by setting the coefficients of all two-electron Slater determinants in Eq. (2) to zero at all times, i.e., the HHG spectra without and with two-electron excited states result from different simulations. The TDKS HHG spectrum without two-electron excited states is calculated by Eq. (3) after removing the coefficients of all two-electron Slater determinants, i.e., it results from the same simulation as the HHG spectrum with two-electron excited states.

It is important to realize that the above findings do not imply that two-electron excited states play no role at all. They alter the populations of the one-electron excited states and, therefore, must be considered to explain the appearance of even-order harmonics in the TDKS HHG spectrum. Figure 2(b) illustrates the possible transitions. First, there are transitions among one-electron excited states and among two-electron excited states (red arrows). Second, there are transitions between the ground state and one-electron excited states, between one-electron excited states and twoelectron excited states, and between the ground state and two-electron excited states (blue arrows). Since the HHG spectrum is determined by one-electron excited states, we are interested in paths leading from the ground state to the one-electron excited states (e.g., $1,1+2+4,1+3+1$ ).
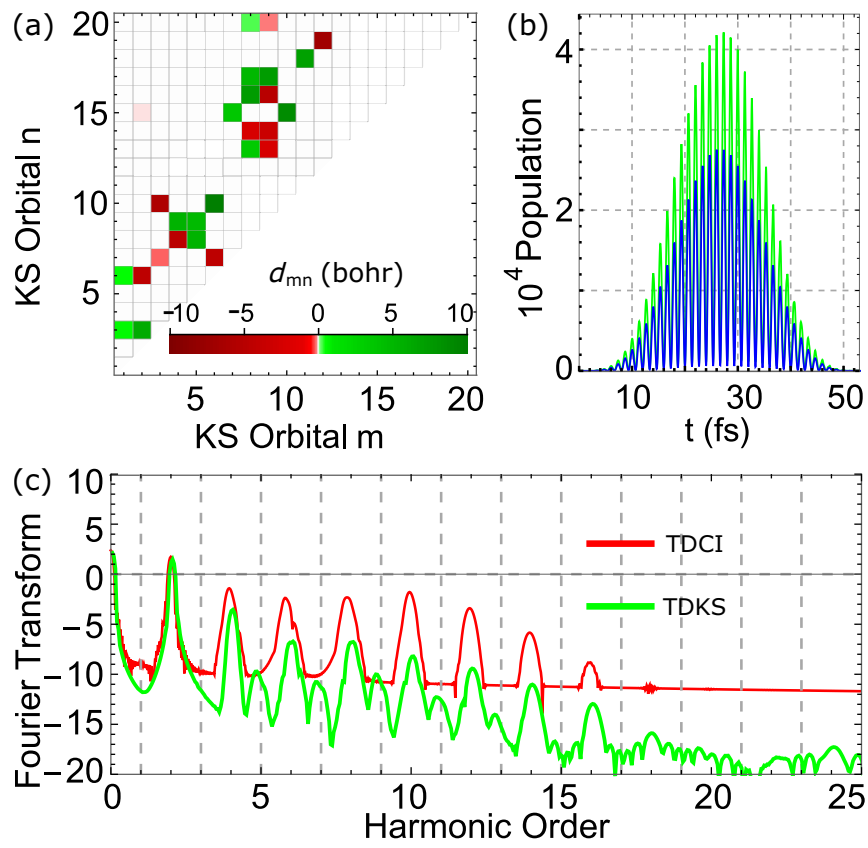

FIG. 3. (a) Transition dipoles of a $\mathrm{H}_{2}$ molecule, showing that $d_{13}$ and $d_{16}$ dominate for transitions from the ground state $(m=1)$. (b) TDKS populations of the dominant one-electron excited states $P_{13}$ (green) and $P_{16}$ (blue), which exceed the populations of all other one-electron excited states by more than three orders of magnitude. (c) TDKS and TDCI Fourier transforms of $P_{13}$.
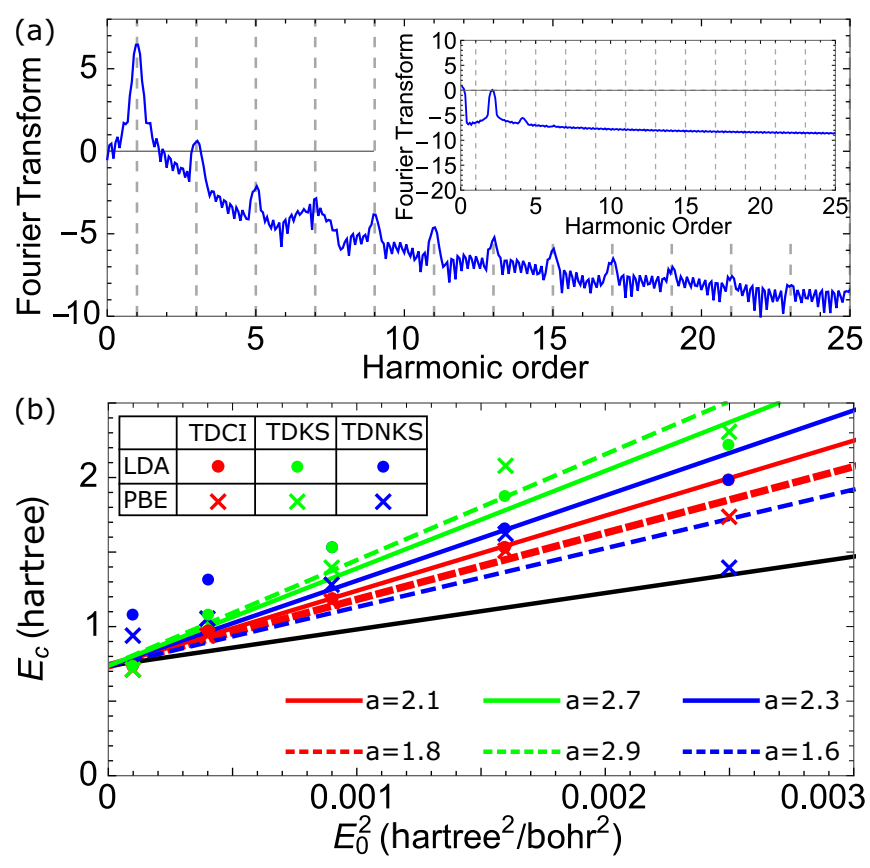

FIG. 4. (a) TDNKS HHG spectrum of a $\mathrm{H}_{2}$ molecule for $\hbar \omega=$ $1.55 \mathrm{eV}, \sigma=1.33 \times 10^{-14} \mathrm{~s}$, and $E_{0}=0.5 \mathrm{~V} / \AA$. Inset: Fourier transform of $P_{13}$. (b) Energy of the spectral cutoff vs laser intensity (data points) with linear fits (colored lines), and Lewenstein model (black line). 

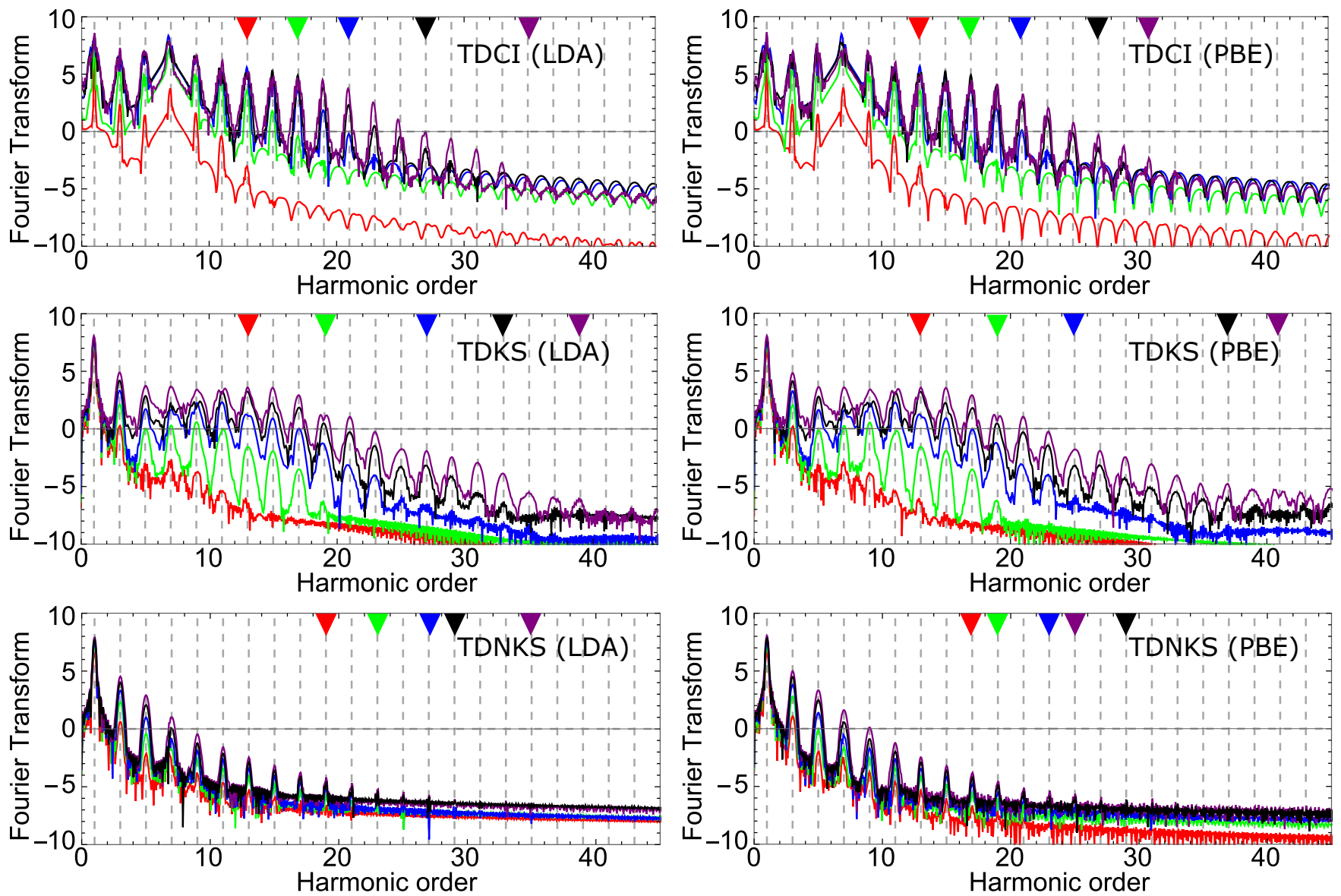

FIG. 5. HHG spectra of a $\mathrm{H}_{2}$ molecule for $\hbar \omega=1.55 \mathrm{eV}, \sigma=1.33 \times 10^{-14} \mathrm{~s}$, and $E_{0}=0.5$ (red), 1.0 (green), 1.5 (blue), 2.0 (black), and 2.5 (purple) $\mathrm{V} / \AA$. The spectral cutoffs are indicated by triangles. The pronounced TDCI peak at harmonic order 7 (10.85 eV) is explained by proximity to the transition energy between the highest occupied and lowest unoccupied molecular orbitals $(10.34 \mathrm{eV})$.

A path consisting of $n$ transitions contributes to the dipole moment proportional to $E(t)^{n}$. Thus, the exponent $n$ is the harmonic order of the dipole moment after Fourier transformation.

Transitions indicated by red arrows in Fig. 2(b) are not to be considered since paths including such transitions give rise to even-order harmonics, and the TDCI HHG spectrum, which is expected to be accurate, does not show even-order harmonics. As for each transition among one-electron excited states there is an inverse transition and these contributions cancel out each other, only the physical transitions indicated by blue arrows in Fig. 2(b) need to be considered. Therefore, we have only odd-order harmonics, consistent with the TDCI HHG spectrum. The transition indicated by a dashed blue arrow in Fig. 2(b) is an unphysical transition (shortcoming of the TDKS equations [52]) that accompanies transition 1 and opens the possibility of even-order harmonics (e.g., path $5+4$ corresponds to a second-order harmonic).

While the unphysical two-electron excited states do not directly modify the HHG spectrum of the $\mathrm{H}_{2}$ molecule, they influence the populations of the one-electron excited states (through paths such as $5+4$ ). We use $M=20 \mathrm{KS}$ orbitals at $t=0$ to expand the time-propagated KS orbitals. Figure 3(a) indicates that there are two dominant transition dipoles, $d_{13}$ and $d_{16}$, for transitions from the ground state $(m=1)$. Their populations $P_{m n}=\left|C_{m n}\right|^{2}$ calculated by the TDKS equations are shown in Fig. 3(b). The TDCI equations give qualitatively the same result. We expect from Fig. 2(b) that $C_{1 n}$ contains only odd powers of $\sin (\omega t)$, and when we consider only $C_{1 n}$ we have $d_{y}(t)=\left[C_{1 n}^{*}(t) C_{11}(t)+C_{11}^{*}(t) C_{1 n}(t)\right] d_{1 n}$. Since the ground state is only weakly affected by the excitation, it can be assumed that $C_{11}$ does not depend on $\sin (\omega t)$. Thus, all observable harmonics must be related to the dynamics of $C_{1 n}$. This is confirmed by Fourier transformation of $P_{13}$ : Clean even-order harmonics are visible in Fig. 3(c) for the TDCI equations. They correspond to the odd powers of $\sin (\omega t)$ in $C_{13}$ and therefore to the odd-order harmonics in the HHG spectrum. The odd-order harmonics visible in Fig. 3(c) for the TDKS equations correspond to the symmetry-forbidden even-order harmonics in the HHG spectrum. The model of Fig. 2(b) to explain the failure of the TDKS equations is thus confirmed.

To further verify that the origin of the symmetry-forbidden harmonics in the TDKS HHG spectrum is the incorrect populations of the one-electron excited states (due to fixation of the number of KS orbitals and their occupations), we employ the TDNKS equations, i.e., the number of KS orbitals and their occupations are updated on the fly so that unphysical two-electron excited states are avoided. To reduce the computational cost, we use a shorter electric-field envelope of $\sigma=1.33 \times 10^{-14} \mathrm{~s}$. The obtained HHG spectrum in Fig. 4(a) 

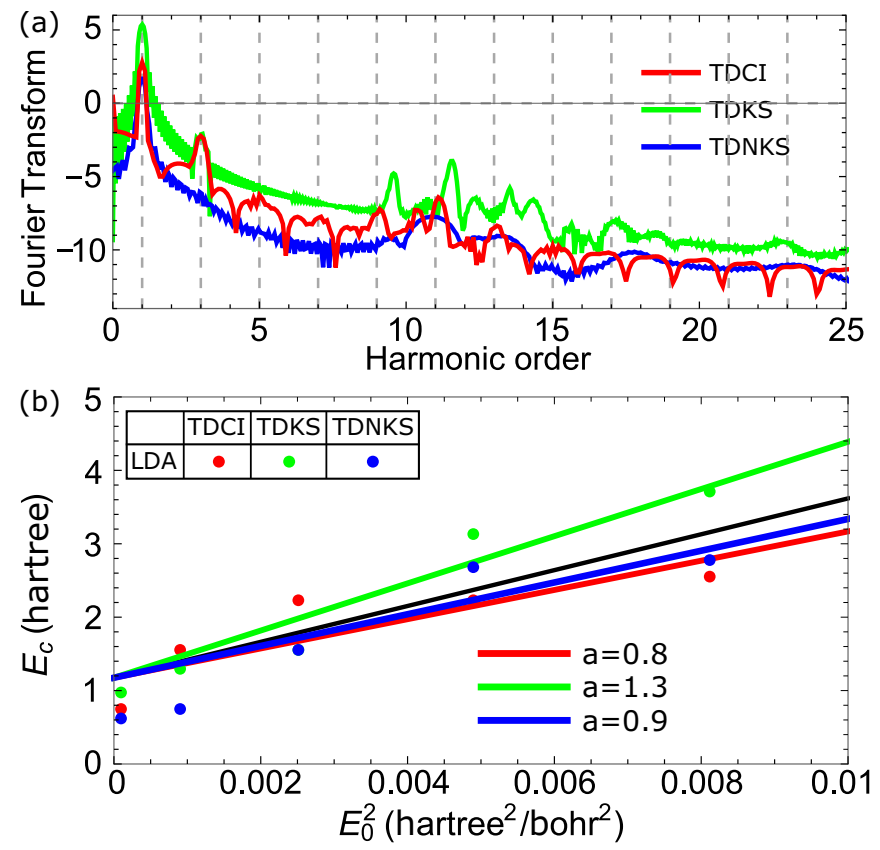

FIG. 6. (a) HHG spectra of a He atom for $\hbar \omega=1.55 \mathrm{eV}, \sigma=$ $1.33 \times 10^{-14} \mathrm{~s}$, and $E_{0}=0.5 \mathrm{~V} / \AA$. (b) Energy of the spectral cutoff vs laser intensity (data points) with linear fits (colored lines), and Lewenstein model (black line).

shows no even-order harmonics and the Fourier transform of $P_{13}$ in the inset shows only even-order harmonics, resembling the TDCI results in Fig. 3(c). Therefore, both the TDCI and TDNKS equations yield clean odd-order harmonics, in contrast to the TDKS equations. The fact that the even-order harmonics become weaker and eventually disappear for increasing laser intensity indicates that transitions from the ground state dominate the populations of the one-electron excited states over transitions from unphysical two-electron excited states.

As the TDKS, TDCI, and TDNKS HHG spectra are rather different, the question arises of how well the TDKS and TDNKS equations perform as compared to the TDCI equations. To answer this question, we compare the energy of the spectral cutoff $\left(E_{c}\right)$ for the three methods using both the LDA and PBE exchange-correlation functionals; see Fig. 5. The spectral cutoff is defined as the highest attainable harmonic order. While it is usually given by a steep drop after a plateau in the HHG spectrum $[5,55]$, for a short laser pulse it is determined as the end of the cutoff region $[8,10]$. Notice that we use low intensity to avoid ionization [56,57]. While the TDCI and TDKS HHG spectra show distinct plateaus, this applies to the TDNKS HHG spectra only at low $E_{0}$. The reason is that the low-order harmonics are enhanced faster than the high-order harmonics for increasing $E_{0}$, which gives rise to an overall monotonic decay of the TDNKS HHG spectra. We also note that for increasing $E_{0}$, the plateaus extend to higher harmonic order in both the TDKS and the TDCI HHG spectra. We fit our data as

$$
E_{c}\left(E_{0}^{2}\right)=1.3 I_{p}+3.17 \frac{a E_{0}^{2}}{4 \omega^{2}},
$$
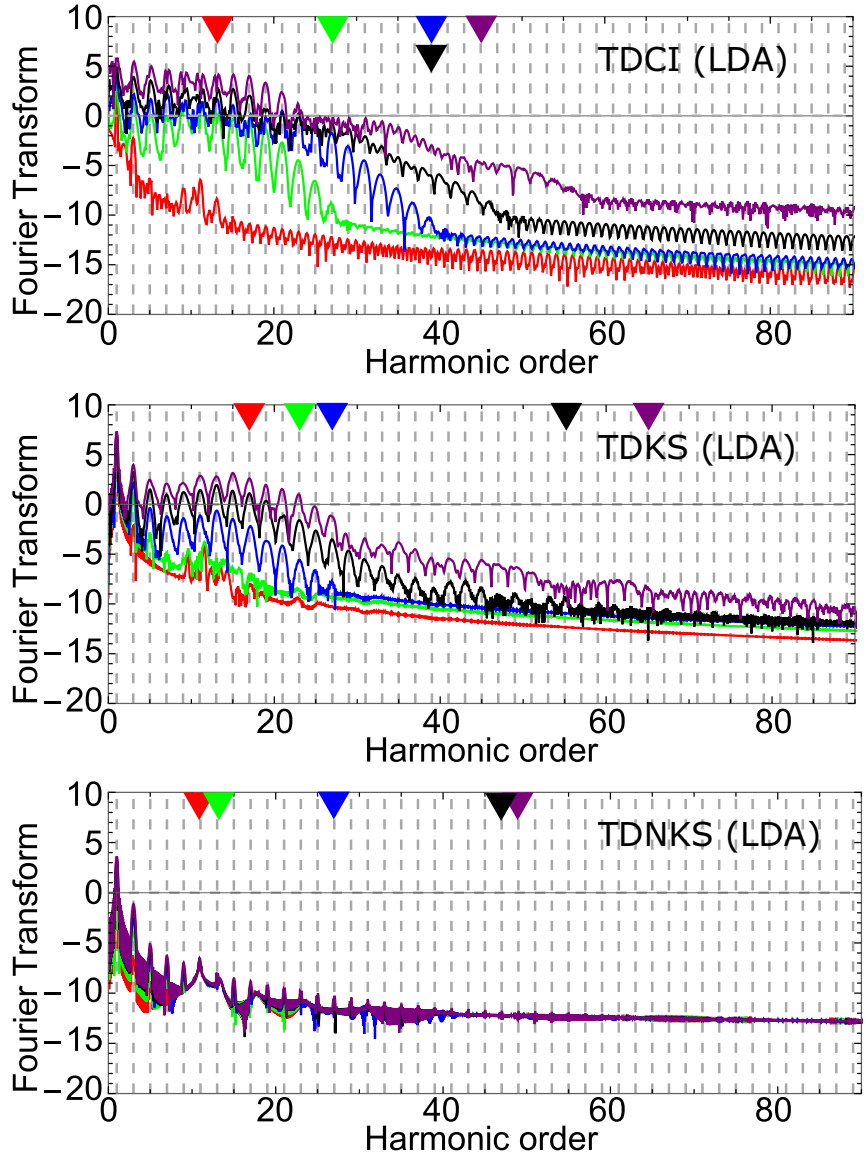

FIG. 7. HHG spectra of a He atom for $\hbar \omega=1.55 \mathrm{eV}, \sigma=$ $1.33 \times 10^{-14} \mathrm{~s}$, and $E_{0}=0.5$ (red), 1.5 (green), 2.5 (blue), 3.5 (black), and 4.5 (purple) $\mathrm{V} / \AA$. The spectral cutoffs are indicated by triangles.

where $I_{p}=15.4 \mathrm{eV}$ is the ionization energy of the $\mathrm{H}_{2}$ molecule. The Lewenstein model, which is more accurate than the three-step model [5], corresponds to $a=1$. Figure 4(b) shows that both the TDCI and TDNKS equations obey the Lewenstein model rather well, in contrast to the TDKS equations. The fact that the TDCI equations are accurate for the KS system and obey the Lewenstein model validates our definition of the spectral cutoff. Note, also, that the spectral cutoff in both the Lewenstein and three-step models, in agreement with our definition, is the maximum energy that an electron can obtain. Clearly, the TDKS equations predict the energy of the spectral cutoff poorly, while the TDNKS equations perform better. Finally, we find that the choice of the exchange-correlation functional (LDA or PBE) hardly influences the HHG spectrum.

To evaluate whether the large static correlation of the $\mathrm{H}_{2}$ molecule affects our conclusions, Figs. 6 and 7 provide TDCI, TDKS, and TDNKS HHG spectra for a He atom (small static correlation [58]). The presence and absence of even-order harmonics in the TDKS and TDNKS HHG spectra, respectively, show that the static correlation is not responsible for the evenorder harmonics. Again, the TDNKS equations predict the energy of the spectral cutoff better than the TDKS equations 
( $I_{p}=24.6 \mathrm{eV}$ for the He atom); see Fig. 6(b). Note that all TDNKS HHG spectra show distinct plateaus.

\section{CONCLUSIONS}

Our results show that the TDKS equations generate symmetry-forbidden harmonics for both a $\mathrm{H}_{2}$ molecule and a $\mathrm{He}$ atom, but not for a $\mathrm{H}$ atom. We demonstrate that this shortcoming is due to incorrect populations of the one-electron excited states, as a consequence of fixation of the number of KS orbitals and their occupations. Interestingly, direct effects of the unphysical two-electron excited states resulting from the TDKS equations turn out to be negligible, while indirect effects on the populations of the one-electron excited states are critical. We also find that the TDKS equations yield a wrong energy of the spectral cutoff. As the TDNKS equations do not share the shortcomings of the TDKS equations, they provide a favorable formulation of real-time time-dependent density functional theory. The development of TDNKS equations for periodic systems and systems with more than two electrons, therefore, appears to be a promising route. We note that no unphysical even-order harmonics have been reported in Refs. [56] and [59] for $E_{0}=2.5$ and $5.0 \mathrm{~V} / \AA$, respectively, due to the fact that they become buried in the enhanced oddorder harmonics at growing laser strength.

\section{ACKNOWLEDGMENTS}

We acknowledge financial support from King Abdullah University of Science and Technology (KAUST). For computer time, this research used the resources of the Supercomputing Laboratory at KAUST. We thank Nicolas Tancogne-Dejean and Aurélien Manchon for helpful discussions.

\section{APPENDIX A}

The TDKS and TDNKS formulations share the equations of motion

$$
\begin{aligned}
i \hbar \frac{\partial}{\partial t}\left|\psi_{m}(t)\right\rangle= & {\left[-\frac{\hbar^{2}}{2 m_{e}} \nabla^{2}+v_{\text {ext }}(t)+v_{\text {Hartree }}[\rho](t)\right.} \\
& \left.+v_{x c}[\rho](t)\right]\left|\psi_{m}(t)\right\rangle,
\end{aligned}
$$

where $\left|\psi_{m}(t)\right\rangle, v_{\text {ext }}, v_{\text {Hartree }}$, and $v_{x c}$ denote the natural KS orbitals, external potential, Hartree potential, and exchangecorrelation potential, respectively. The spin-reduced electron density is constructed from $M(t)$ natural KS orbitals with occupations $p_{m}(t)$ as $\rho(t)=\sum_{m=1}^{M(t)} p_{m}(t)\left|\psi_{m}(t)\right|^{2}$ and the time-propagated wave function can be written as

$$
|\Psi(t)\rangle=\sum_{m, n=1}^{M} C_{m n}(t)\left|\psi_{m} \bar{\psi}_{n}\right\rangle,
$$

with $\left|\psi_{m}\right\rangle=\left|\psi_{m}(t=0)\right\rangle$. Removing two-electron excitations from Eq. (A2), we obtain

$$
\mathbf{C}=\frac{1}{N_{C}}\left(\begin{array}{cccc}
C_{11} & C_{12} & \cdots & C_{1 M} \\
C_{21} & 0 & \cdots & 0 \\
\vdots & \vdots & \ddots & \vdots \\
C_{M 1} & 0 & \cdots & 0
\end{array}\right)
$$

TABLE I. Parameters of the applied Tukey windows.

\begin{tabular}{lcccc}
\hline \hline System & \multicolumn{2}{c}{$\mathrm{H}_{2}$} & $\mathrm{He}$ & $\mathrm{H}$ \\
\hline Laser duration & $26.7 \mathrm{fs}$ & $13.3 \mathrm{fs}$ & $13.3 \mathrm{fs}$ & $26.7 \mathrm{fs}$ \\
TDCI & $\Delta t=52 \mathrm{fs}$ & & $\Delta t=26 \mathrm{fs}$ & \\
& $t_{0}=26 \mathrm{fs}$ & & $t_{0}=13 \mathrm{fs}$ & \\
& $\alpha=0.07$ & & $\alpha=0.12$ & \\
TDKS & $\Delta t=52 \mathrm{fs}$ & $\Delta t=30 \mathrm{fs}$ & $\Delta t=30 \mathrm{fs}$ & $\Delta t=52 \mathrm{fs}$ \\
& $t_{0}=26 \mathrm{fs}$ & $t_{0}=15 \mathrm{fs}$ & $t_{0}=15 \mathrm{fs}$ & $t_{0}=26 \mathrm{fs}$ \\
& $\alpha=0.20$ & $\alpha=0.01$ & $\alpha=0.01$ & $\alpha=0.20$ \\
TDNKS & & $\Delta t=30 \mathrm{fs}$ & $\Delta t=30 \mathrm{fs}$ & \\
& & $t_{0}=15 \mathrm{fs}$ & $t_{0}=15 \mathrm{fs}$ & \\
& & $\alpha=0.10$ & $\alpha=0.10$ & \\
\hline \hline
\end{tabular}

with the renormalization factor $N_{C}=\sqrt{\sum_{m, n=1}^{M}\left|C_{m n}\right|^{2}}$. The corresponding density matrix is $\rho=\mathbf{C C}^{\dagger}$ and diagonalization $\mathbf{D}=\mathbf{U}^{\dagger} \rho \mathbf{U}$ provides the updated natural $\mathrm{KS}$ orbitals $\left|\psi_{m}(t)\right\rangle=\sum_{n=1}^{M}\left|\psi_{n}\right\rangle U_{n m}(t)$ and their occupations $2 D_{m m}$. The updated wave function is

$$
|\Psi(t)\rangle=\sum_{m, n=1}^{M} B_{m n}\left|\psi_{m}(t) \bar{\psi}_{n}(t)\right\rangle
$$

with $\mathbf{B}=\mathbf{U}^{*} \mathbf{C} \mathbf{U}^{\dagger}$. The updated natural KS orbitals are propagated by Eq. (A1) and the procedure is iterated.

\section{APPENDIX B}

The parameters of the applied Tukey windows

$$
W_{T}(t)=\left\{\begin{array}{l}
\frac{1+\cos \left[\pi\left(2 \frac{t-t_{0}}{\Delta t \alpha}-1+\frac{1}{\alpha}\right)-\phi\right]}{2} \text { for } \frac{-1}{2} \leqslant \frac{t-t_{0}}{\Delta t}<\frac{\alpha-1}{2} \\
1 \text { for } \frac{\alpha-1}{2} \leqslant \frac{t-t_{0}}{\Delta t} \leqslant \frac{1-\alpha}{2} \\
\frac{1+\cos \left[\pi\left(2 \frac{t-t_{0}}{\Delta t \alpha}+1-\frac{1}{\alpha}\right)-\phi\right]}{2} \text { for } \frac{1-\alpha}{2}<\frac{t-t_{0}}{\Delta t} \leqslant \frac{1}{2} \\
0 \text { otherwise, }
\end{array}\right.
$$

with $0<\alpha<1$, are given in Table I.

\section{APPENDIX C}

The $\mathrm{H}_{2}$ molecule is modeled as a two-electron $\mathrm{KS}$ system with Hamiltonian

$$
H_{0}=\sum_{i}^{N}\left[-\frac{1}{2} \nabla_{i}^{2}+v_{K S}\left(\vec{r}_{i}\right)\right]=\sum_{i}^{N} h_{K S}\left(\vec{r}_{i}\right),
$$

where $v_{K S}$ is the KS potential, $N$ is the number of electrons, and $h_{K S}$ is the KS Hamiltonian. Including the external electric field in the dipole approximation, the total Hamiltonian is

$$
H=H_{0}+\sum_{i=1}^{N} \vec{r}_{i} \cdot \vec{E}(t) .
$$

The initial state is the ground state with the wave function given by the Slater determinant

$$
|\Psi(t=0)\rangle=\left|\psi_{1} \bar{\psi}_{1}\right\rangle \equiv \frac{1}{\sqrt{2}}\left|\begin{array}{ll}
\psi_{1}\left(\vec{r}_{1}\right) & \bar{\psi}_{1}\left(\vec{r}_{1}\right) \\
\psi_{1}\left(\vec{r}_{2}\right) & \bar{\psi}_{1}\left(\vec{r}_{2}\right)
\end{array}\right|,
$$


where $\left|\psi_{m}\right\rangle$ are the KS orbitals satisfying the KS equation

$$
h_{K S}\left|\psi_{m}\right\rangle=\varepsilon_{m}\left|\psi_{m}\right\rangle \text {, }
$$

with eigenenergies $\varepsilon_{m}$. We obtain the time-propagated multielectron wave function by solving the time-dependent Schrödinger equation (atomic units),

$$
i \frac{d}{d t}|\Psi(t)\rangle=H|\Psi(t)\rangle
$$

through the TDCI or TDKS equations.

In the case of the TDCI equations, expansion of $|\Psi(t)\rangle$ in the basis of multielectron wave functions of $H_{0}$ [Eq. (A2)] and substitution into Eq. (C5) yields

$$
\begin{gathered}
i \sum_{m^{\prime}, n^{\prime}=1}^{M} \frac{\partial C_{m^{\prime} n^{\prime}}(t)}{\partial t}\left|\psi_{m^{\prime}} \psi_{n^{\prime}}\right\rangle=\sum_{m^{\prime}, n^{\prime}=1}^{M} C_{m^{\prime} n^{\prime}}(t) \\
\times\left(H_{0}+\sum_{i}^{N} \vec{r}_{i} \cdot \vec{E}(t)\right)\left|\psi_{m^{\prime}} \psi_{n^{\prime}}\right\rangle .
\end{gathered}
$$

Noting that

$$
\begin{aligned}
\left\langle\psi_{m}\right. & \bar{\psi}_{n}\left|\psi_{m^{\prime}} \bar{\psi}_{n^{\prime}}\right\rangle \\
= & \frac{1}{2}\left(\left\langle\psi_{m}\left(\vec{r}_{1}\right)\right|\left\langle\bar{\psi}_{n}\left(\vec{r}_{2}\right)\right|-\left\langle\psi_{m}\left(\vec{r}_{2}\right)\right|\left\langle\bar{\psi}_{n}\left(\vec{r}_{1}\right)\right|\right) \\
& \times\left(\left|\psi_{m^{\prime}}\left(\vec{r}_{1}\right)\right\rangle\left|\bar{\psi}_{n^{\prime}}\left(\vec{r}_{2}\right)\right\rangle-\left|\psi_{m^{\prime}}\left(\vec{r}_{2}\right)\right\rangle\left|\bar{\psi}_{n^{\prime}}\left(\vec{r}_{1}\right)\right\rangle\right) \\
= & \delta_{m m^{\prime}} \delta_{n n^{\prime}}
\end{aligned}
$$

implies

$$
\begin{aligned}
& \left\langle\psi_{m} \bar{\psi}_{n}\left|H_{0}\right| \psi_{m^{\prime}} \bar{\psi}_{n^{\prime}}\right\rangle \\
& =\frac{1}{2}\left(\left\langle\psi_{m}\left(\vec{r}_{1}\right)\right|\left\langle\bar{\psi}_{n}\left(\vec{r}_{2}\right)\right|-\left\langle\psi_{m}\left(\vec{r}_{2}\right)\right|\left\langle\bar{\psi}_{n}\left(\vec{r}_{1}\right)\right|\right) \\
& \quad \times \sum_{i=1}^{2} h_{K S}\left(\vec{r}_{i}\right)\left(\left|\psi_{m^{\prime}}\left(\vec{r}_{1}\right)\right\rangle\left|\bar{\psi}_{n^{\prime}}\left(\vec{r}_{2}\right)\right\rangle-\left|\psi_{m^{\prime}}\left(\vec{r}_{2}\right)\right\rangle\left|\bar{\psi}_{n^{\prime}}\left(\vec{r}_{1}\right)\right\rangle\right) \\
& =\left(\varepsilon_{m}+\varepsilon_{n}\right) \delta_{m m^{\prime}} \delta_{n n^{\prime}},
\end{aligned}
$$

and assuming that $\vec{E}(t)$ is polarized along $\hat{y}$ leads to

$$
\begin{aligned}
& \left\langle\psi_{m} \bar{\psi}_{n}\left|\sum_{i=1}^{2} \vec{r}_{i} \cdot \vec{E}(t)\right| \psi_{m^{\prime}} \bar{\psi}_{n^{\prime}}\right\rangle \\
& =\left\langle\psi_{m} \bar{\psi}_{n}\left|\sum_{i=1}^{2} y_{i} \cdot E(t)\right| \psi_{m^{\prime}} \bar{\psi}_{n^{\prime}}\right\rangle \\
& =\left(d_{m m^{\prime}} \delta_{n n^{\prime}}+d_{n n^{\prime}} \delta_{m m^{\prime}}\right) E(t) .
\end{aligned}
$$

Multiplying Eq. (C6) from the left by $\left\langle\psi_{m} \bar{\psi}_{n}\right|$ and employing Eqs. (C7)-(C9), we obtain

$$
\begin{aligned}
& i \frac{\partial}{\partial t} C_{m n}(t)=\left(\varepsilon_{m}+\varepsilon_{n}\right) C_{m n}(t) \\
& +\sum_{m^{\prime}=1}^{M} C_{m^{\prime} n}(t) d_{m m^{\prime}}+\sum_{n^{\prime}=1}^{M} C_{m n^{\prime}}(t) d_{n n^{\prime}},
\end{aligned}
$$

where the eigenenergies $\varepsilon_{m}$ and transition dipoles $d_{m m^{\prime}}$ can be calculated by density functional theory. The dipole moment is given by

$$
\begin{aligned}
d_{y}(t) \equiv & \left\langle\Psi(t)\left|y_{1}+y_{2}\right| \Psi(t)\right\rangle \\
= & \sum_{n=1}^{M} \sum_{m^{\prime}, m=1}^{M} C_{m^{\prime} n}^{*}(t) C_{m n}(t) d_{m^{\prime} m} \\
& +\sum_{m=1}^{M} \sum_{n^{\prime}, n=1}^{M} C_{m n^{\prime}}^{*}(t) C_{m n}(t) d_{n^{\prime} n} .
\end{aligned}
$$

Neglecting the two-electron excited states, Eq. (C11) simplifies to

$$
\begin{aligned}
d_{y}(t)= & \sum_{m^{\prime}, m=1}^{M} C_{m^{\prime} 1}^{*}(t) C_{m 1}(t) d_{m^{\prime} m} \\
& +\sum_{n^{\prime}, n=1}^{M} C_{1 n^{\prime}}^{*}(t) C_{1 n}(t) d_{n^{\prime} n} .
\end{aligned}
$$

Propagating the KS orbitals by the TDKS equations,

$$
i \frac{\partial}{\partial t}\left|\psi_{i}(t)\right\rangle=h_{K S}[\rho]\left|\psi_{i}(t)\right\rangle,
$$

where $\rho=\sum_{i=1}^{N}\left|\psi_{i}(t)\right\rangle\left\langle\psi_{i}(t)\right|$, results in the time-propagated multielectron wave function $|\Psi(t)\rangle=\left|\psi_{1}(t) \bar{\psi}_{1}(t)\right\rangle$. The dipole moment is obtained as

$$
d_{y}(t)=2\left\langle\psi_{1}(t)|y| \psi_{1}(t)\right\rangle,
$$

where the factor two represents the two electrons in the lowest orbital. The TDNKS and TDKS formulations share the time propagation, being distinguished only by the calculation of the electron density. In the TDNKS formulation, we have

$$
d_{y}(t)=\sum_{m=1}^{M(t)} p_{m}(t)\left\langle\psi_{m}(t)|y| \psi_{m}(t)\right\rangle \text {. }
$$

[1] N. H. Burnett, H. A. Baldis, M. C. Richardson, and G. D. Enright, Harmonic Generation in $\mathrm{CO}_{2}$ Laser Target Interaction, Appl. Phys. Lett. 31, 172 (1977).

[2] C. Winterfeldt, C. Spielmann, and G. Gerber, Colloquium: Optimal control of high-harmonic generation, Rev. Mod. Phys. 80, 117 (2008).

[3] K. J. Schafer, B. Yang, L. F. DiMauro, and K. C. Kulander, Above Threshold Ionization Beyond the High Harmonic Cutoff, Phys. Rev. Lett. 70, 1599 (1993).

[4] P. B. Corkum, Plasma Perspective on Strong-Field Multiphoton Ionization, Phys. Rev. Lett. 71, 1994 (1993).
[5] M. Lewenstein, P. Balcou, M. Y. Ivanov, A. L'Huillier, and P. B. Corkum, Theory of high-harmonic generation by lowfrequency laser fields, Phys. Rev. A 49, 2117 (1994).

[6] Z. Chang, A. Rundquist, H. Wang, M. M. Murnane, and H. C. Kapteyn, Generation of Coherent Soft X Rays at $2.7 \mathrm{~nm}$ Using High Harmonics, Phys. Rev. Lett. 79, 2967 (1997).

[7] C. Spielmann, N. H. Burnett, S. Sartania, R. Koppitsch, M. Schnürer, C. Kan, M. Lenzner, P. Wobrauschek, and F. Krausz, Generation of coherent X-rays in the water window using 5-femtosecond laser pulses, Science 278, 661 (1997). 
[8] I. P. Christov, M. M. Murnane, and H. C. Kapteyn, Highharmonic Generation of Attosecond Pulses in the "SingleCycle" Regime, Phys. Rev. Lett. 78, 1251 (1997).

[9] X. Ren, J. Li, Y. Yin, K. Zhao, A. Chew, Y. Wang, S. Hu, Y. Cheng, E. Cunningham, Y. Wu, M. Chini, and Z. Chang, Attosecond light sources in the water window, J. Opt. 20, 023001 (2018).

[10] B. Shan and Z. Chang, Dramatic extension of the high-order harmonic cutoff by using a long-wavelength driving field, Phys. Rev. A 65, 011804(R) (2001).

[11] E. J. Takahashi, T. Kanai, K. L. Ishikawa, Y. Nabekawa, and K. Midorikawa, Coherent Water Window X Ray by PhaseMatched High-Order Harmonic Generation in Neutral Media, Phys. Rev. Lett. 101, 253901 (2008).

[12] H. Xiong, H. Xu, Y. Fu, J. Yao, B. Zeng, W. Chu, Y. Cheng, Z. Xu, E. J. Takahashi, K. Midorikawa, X. Liu, and J. Chen, Generation of a coherent $X$ ray in the water window region at $1 \mathrm{kHz}$ repetition rate using a mid-infrared pump source, Opt. Lett. 34, 1747 (2009).

[13] M.-C. Chen, P. Arpin, T. Popmintchev, M. Gerrity, B. Zhang, M. Seaberg, D. Popmintchev, M. M. Murnane, and H. C. Kapteyn, Bright, Coherent, Ultrafast Soft X-Ray Harmonics Spanning the Water Window From a Tabletop Light Source, Phys. Rev. Lett. 105, 173901 (2010).

[14] N. Ishii, K. Kaneshima, K. Kitano, T. Kanai, S. Watanabe, and J. Itatani, Carrier-envelope phase-dependent high harmonic generation in the water window using few-cycle infrared pulses, Nat. Commun. 5, 3331 (2014).

[15] A. Johnson, L. Miseikis, D. Wood, D. Austin, C. Brahms, S. Jarosch, C. Strüber, P. Ye, and J. Marangos, Measurement of sulfur $\mathrm{L}_{2,3}$ and carbon $\mathrm{K}$ edge XANES in a polythiophene film using a high harmonic supercontinuum, Struct. Dyn. 3, 062603 (2016).

[16] S. Teichmann, F. Silva, S. Cousin, M. Hemmer, and J. Biegert, 0.5-keV soft x-ray attosecond continua, Nat. Commun. 7, 11493 (2016).

[17] G. J. Stein, P. D. Keathley, P. Krogen, H. Liang, J. P. Siqueira, C.-L. Chang, C.-J. Lai, K.-H. Hong, G. M. Laurent, and F. X. Kärtner, Water-window soft x-ray high-harmonic generation up to the nitrogen $\mathrm{K}$-edge driven by a $\mathrm{kHz}, 2.1 \mu \mathrm{m}$ OPCPA source, J. Phys. B: At. Mol. Opt. Phys. 49, 155601 (2016).

[18] T. Popmintchev, M. Chen, D. Popmintchev, P. Arpin, S. Brown, S. Ališauskas, G. Andriukaitis, T. Balčiunas, O. D. Mücke, A. Pugzlys, A. Baltuška, B. Shim, S. E. Schrauth, A. Gaeta, C. Hernández-García, L. Plaja, A. Becker, A. Jaron-Becker, M. M. Murnane, and H. C. Kapteyn, Bright coherent ultrahigh harmonics in the keV X-ray regime from mid-infrared femtosecond lasers, Science 336, 1287 (2012).

[19] S. Ghimire, A. D. DiChiara, E. Sistrunk, P. Agostini, L. F. DiMauro, and D. A. Reis, Observation of high-order harmonic generation in a bulk crystal, Nat. Phys. 7, 138 (2011).

[20] O. Schubert, M. Hohenleutner, F. Langer, B. Urbanek, C. Lange, U. Huttner, D. Golde, T. Meier, M. Kira, S. W. Koch, and R. Huber, Sub-cycle control of terahertz high-harmonic generation by dynamical Bloch oscillations, Nat. Photon. 8, 119 (2014).

[21] M. Hohenleutner, F. Langer, O. Schubert, M. Knorr, U. Huttner, S. W. Koch, M. Kira, and R. Huber, Real-time observation of interfering crystal electrons in high-harmonic generation, Nature (London) 523, 572 (2015).
[22] T. T. Luu, M. Garg, S. Y. Kruchinin, A. Moulet, M. T. Hassan, and E. Goulielmakis, Extreme ultraviolet high-harmonic spectroscopy of solids, Nature (London) 521, 498 (2015).

[23] G. Ndabashimiye, S. Ghimire, M. Wu, D. A. Browne, K. J. Schafer, M. B. Gaarde, and D. A. Reis, Solid-state harmonics beyond the atomic limit, Nature (London) 534, 520 (2016).

[24] D. Golde, T. Meier, and S. W. Koch, High harmonics generated in semiconductor nanostructures by the coupled dynamics of optical inter- and intraband excitations, Phys. Rev. B 77, 075330 (2008).

[25] D. Golde, T. Meier, and S. W. Koch, Microscopic analysis of high-harmonic generation in semiconductor nanostructures, Phys. Status Solidi C 6, 420 (2009).

[26] D. Golde, M. Kira, T. Meier, and S. W. Koch, Microscopic theory of the extremely nonlinear terahertz response of semiconductors, Phys. Status Solidi B 248, 863 (2011).

[27] O. D. Mücke, Isolated high-order harmonics pulse from twocolor-driven Bloch oscillations in bulk semiconductors, Phys. Rev. B 84, 081202(R) (2011).

[28] P. G. Hawkins, M. Y. Ivanov, and V. S. Yakovlev, Effect of multiple conduction bands on high-harmonic emission from dielectrics, Phys. Rev. A 91, 013405 (2015).

[29] M. Wu, S. Ghimire, D. A. Reis, K. J. Schafer, and M. B. Gaarde, High-harmonic generation from Bloch electrons in solids, Phys. Rev. A 91, 043839 (2015).

[30] T. Otobe, High-harmonic generation in $\alpha$-quartz by electronhole recombination, Phys. Rev. B 94, 235152 (2016).

[31] T. T. Luu and H. J. Wörner, High-order harmonic generation in solids: A unifying approach, Phys. Rev. B 94, 115164 (2016).

[32] N. Tancogne-Dejean, O. D. Mücke, F. X. Kärtner, and A. Rubio, Impact of the Electronic Band Structure in High-Harmonic Generation Spectra of Solids, Phys. Rev. Lett. 118, 087403 (2017).

[33] R. Haight and P. F. Seidler, High resolution atomic core level spectroscopy with laser harmonics, Appl. Phys. Lett. 65, 517 (1994).

[34] W. Theobald, R. Häßner, C. Wülker, and R. Sauerbrey, Temporally Resolved Measurement of Electron Densities $\left(>10^{23} \mathrm{~cm}^{-3}\right.$ ) with High Harmonics, Phys. Rev. Lett. 77, 298 (1996).

[35] M. Schnürer, C. Streli, P. Wobrauschek, M. Hentschel, R. Kienberger, C. Spielmann, and F. Krausz, Femtosecond X-Ray Fluorescence, Phys. Rev. Lett. 85, 3392 (2000).

[36] P. M. Paul, E. S. Toma, P. Breger, G. Mullot, F. Augé, P. Balcou, H. G. Muller, and P. Agostini, Observation of a train of attosecond pulses from high harmonic generation, Science 292, 1689 (2001)

[37] P. Tzallas, D. Charalambidis, N. A. Papadogiannis, K. Witte, and G. D. Tsakiris, Direct observation of attosecond light bunching, Nature (London) 426, 267 (2003).

[38] R. Kienberger, E. Goulielmakis, M. Uiberacker, A. Baltuska, V. Yakovlev, F. Bammer, A. Scrinzi, T. Westerwalbesloh, U. Kleineberg, U. Heinzmann, M. Drescher, and F. Krausz, Atomic transient recorder, Nature (London) 427, 817 (2004).

[39] Y. Nabekawa, T. Shimizu, T. Okino, K. Furusawa, H. Hasegawa, K. Yamanouchi, and K. Midorikawa, Interferometric Autocorrelation of an Attosecond Pulse Train in the Single-Cycle Regime, Phys. Rev. Lett. 97, 153904 (2006).

[40] A. J. Uzan, H. Soifer, O. Pedatzur, A. Clergerie, S. Larroque, B. D. Bruner, B. Pons, M. Ivanov, O. Smirnova, and N. 
Dudovich, Spatial molecular interferometry via multidimensional high-harmonic spectroscopy, Nat. Photon. 14, 188 (2020).

[41] S. Han, L. Ortmann, H. Kim, Y. Kim, T. Oka, A. Chacon, B. Doran, M. Ciappina, M. Lewenstein, S. Kim, S. Kim, and A. S. Landsman, Extraction of higher-order nonlinear electronic response in solids using high harmonic generation, Nat. Commun. 10, 3272 (2019).

[42] T. T. Luu and H. J. Wörner, Measurement of the Berry curvature of solids using high-harmonic spectroscopy, Nat. Commun. 9, 916 (2018).

[43] D. Bauer and K. K. Hansen, High-Harmonic Generation in Solids with and without Topological Edge States, Phys. Rev. Lett. 120, 177401 (2018)

[44] A. Chacón, W. Zhu, S. P. Kelly, A. Dauphin, E. Pisanty, D. Kim, D. E. Kim, A. Picón, C. Ticknor, M. F. Ciappina, A. Saxena, and M. Lewenstein, Observing topological phase transitions with high harmonic generation, Phys. Rev. B 102, 134115 (2020).

[45] H. Drüeke and D. Bauer, Robustness of topologically sensitive harmonic generation in laser-driven linear chains, Phys. Rev. A 99, 053402 (2019).

[46] C. Jürß and D. Bauer, High-harmonic generation in SuSchrieffer-Heeger chains, Phys. Rev. B 99, 195428 (2019).

[47] C. Yu, S. Jiang, and R. Lu, High order harmonic generation in solids: A review on recent numerical methods, Adv. Phys. X 4, 1562982 (2019).

[48] T. Tamaya, A. Ishikawa, T. Ogawa, and K. Tanaka, Diabatic Mechanisms of Higher-Order Harmonic Generation in SolidState Materials under High-Intensity Electric Fields, Phys. Rev. Lett. 116, 016601 (2016).

[49] A. F. Kemper, B. Moritz, J. K. Freericks, and T. P. Devereaux, Theoretical description of high-order harmonic generation in solids, New J. Phys. 15, 023003 (2013).

[50] U. Huttner, K. Schuh, J. V. Moloney, and S. W. Koch, Similarities and differences between high-harmonic generation in atoms and solids, J. Opt. Soc. Am. B 33, C22 (2016).
[51] G. Vampa, C. R. McDonald, G. Orlando, D. D. Klug, P. B. Corkum, and T. Brabec, Theoretical Analysis of HighHarmonic Generation in Solids, Phys. Rev. Lett. 113, 073901 (2014)

[52] X. Zang, U. Schwingenschlögl, and M. T. Lusk, Identification and Resolution of Unphysical Multielectron Excitations in the Real-Time Time-Dependent Kohn-Sham Formulation, Phys. Rev. Lett. 124, 026402 (2020).

[53] X. Andrade, D. Strubbe, U. D. Giovannini, A. H. Larsen, M. J. T. Oliveira, J. Alberdi-Rodriguez, A. Varas, I. Theophilou, N. Helbig, M. J. Verstraete, L. Stella, F. Nogueira, A. AspuruGuzik, A. Castro, M. A. L. Marques, and A. Rubio, Real-space grids and the OCTOPUS code as tools for the development of new simulation approaches for electronic systems, Phys. Chem. Chem. Phys. 17, 31371 (2015).

[54] C. F. Craig, W. R. Duncan, and O. V. Prezhdo, Trajectory Surface Hopping in the Time-Dependent Kohn-Sham Approach for Electron-Nuclear Dynamics, Phys. Rev. Lett. 95, 163001 (2005).

[55] J. L. Krause, K. J. Schafer, and K. C. Kulander, High-Order Harmonic Generation from Atoms and Ions in the High Intensity Regime, Phys. Rev. Lett. 68, 3535 (1992).

[56] E. Luppi and M. Head-Gordon, Computation of high-harmonic generation spectra of $\mathrm{H}_{2}$ and $\mathrm{N}_{2}$ in intense laser pulses using quantum chemistry methods and time-dependent density functional theory, Mol. Phys. 110, 909 (2012).

[57] M. Brics, J. Rapp, and D. Bauer, Strong-field absorption and emission of radiation in two-electron systems calculated with time-dependent natural orbitals, Phys. Rev. A 93, 013404 (2016)

[58] C. L. Benavides-Riveros, N. N. Lathiotakis, and M. A. L. Marques, Towards a formal definition of static and dynamic electronic correlations, Phys. Chem. Chem. Phys. 19, 12655 (2017).

[59] X. Chu and G. C. Groenenboom, Time-dependent densityfunctional-theory calculation of high-order-harmonic generation of $\mathrm{H}_{2}$, Phys. Rev. A 85, 053402 (2012). 